\title{
Experimentelle und theoretische Untersuchung des zwischenmolekularen Übergangs von Elektronenanregungsenergie
}

\author{
Von THEOdOR FörSTER \\ Aus dem Max-Planck-Institut für physikalische Chemie, Göttingen \\ (Z. Naturforschg. 4 a, 321-327 [1949]; eingegangen am 13. Januar 1949)
}

\begin{abstract}
Im Anschluß an frühere orientierende Messungen wird die wahre Löschwirkung von Rhodamin B auf die Fluoreszenz von Trypaflavin in Methanol untersucht, indem diese von der durch Absorption bedingten Fluoreszenzschwächung experimentell getrennt wird. Es' wird eine Theorie der Löschung durch zwischenmolekularen Energieübergang entwickelt und mit den Versuchsergebnissen verglichen.
\end{abstract}

$I^{\prime}$ n einer früheren Veröffentlichung ${ }^{1}$ wurde über die experimentelle Untersuchung eines bisher wenig beachteten Typus von Fluoreszenzlöschung berichtet. Dieser liegt dann vor, wenn der Lösung eines fluoreszierenden Stoffes A ein anderer Stoff B zugesetzt wird, der bei längeren Wellen als der erste absorbiert. Die dabei auftretende Verringerung der Fluoreszenzintensität von A geschieht dabei außer durch Absorption des erregenden Lichtes und des Fluoreszenzlichtes durch den zugesetzten Stoff B auch durch wahre Löschung, die auf unmittelbarer Wechselwirkung der Moleküle von A und B beruht. Falls der Stoff B selbst fluoreszenzfähig ist, tritt dabei dessen sensibilisierte Fluoreszenz auf. Mit Trypáflavin als Stoff A und Rhodamin $B$ als Stoff B ergab sich für die wahre Löschung in Methanol eine Halbwertskonzentration von etwa $1 \cdot 10^{-3} \mathrm{Mol} / l$. Selbstverständlich ist dies eine Fluoreszenzlöschung nur insofern, als die Beobachtung auf den Spektralbereich der Trypaflavinfluoreszenz beschränkt bleibt. Die indirekt festgestellte Verringerung der Abklingdauer der Trypaflavinfluoreszenz bei der Löschung zeigt, daß diese nicht statisch durch Assoziation $\mathrm{zu}$ einer fluoreszenzunfähigen Molekülverbindung, sondern dynamisch durch einen molekularen Prozeß folgender Art zu deuten ist:

$\mathrm{A}+\mathrm{B}+\stackrel{\prime}{h} \rightarrow \mathrm{A}^{*}+\mathrm{B} \rightarrow \mathrm{A}+\mathrm{B}^{*} \rightarrow \mathrm{A}+\mathrm{B}+h v^{\prime}$

Dabei ist eine gegenseitige Annäherung der Moleküle duroh Diffusion, wie sie in anderen Fällen von Fluoreszenzlöschung stattfindet, wegen der nur geringen Abhängigkeit von Temperatur und

1 Th. Förster, Z. Elektrochem. 53, 93 [1949]; vgl. auch Angew. Chem. 59, 181 [1947]; 60, 163 [1948].
Lösungsmittelzähigkeit nicht anzunehmen. Der Übergang der Anregungsenergie muß vielmehr über die zwischenliegenden Lösungsmittelmoleküle hinweg auf eine Entfernung von vielen Moleküldurchmessern geschehen.

Wenn auch in den bereits vorliegenden Versuchen die Existenz der wahren Löschung sichergestellt werden konnte, so war deren quantitative Trennung von der durch Absorption vorgetäuschten Löschung doch unvollkommen. Da so die genaue Feststellung der Größe der Löschung, insbesondere bei geringen Konzentrationen, schwierig war, erschien die Weiterführung der Versuche mit einer verbesserten Methode erwünscht. Es wurde dazu eine solche benutzt, die bereits von J. Perrin und Choucroun ${ }^{2}$ bei einer orientierenden Untersuchung dieser Art von Fluoreszenzlöschung angewandt wurde. Sie beruht auf einem Vergleich der Fluoreszenzintensitäten verschieden konzentrierter Mischlösungen beider Stoffe in gleich absorbierenden Schichten. Bei Gültigkeit des Beerschen Absorptionsgesetzes werden dazu die Schichtdicken umgekehrt proportional den Konzentrationen beider Stoffe in den verschiedenen Lösungen gewählt. Es ist dann bei parallelem Erregungslicht der Einfluß der Absorption auf ein paralleles Bündel des Fluoreszenzlichtes der gleiche. Die relative Veränderung der gemessenen Fluoreszenzintensität $I$ ist in diesem Falle gleich derjenigen der inneren Fluoreszenzausbeute $\boldsymbol{\eta}$ und ergibt somit die wahre Löschung.

Für die Messungen wurden wieder die Farbstoffe Trypaflavin und Rhodamin B in Methanollösungen benutzt:

2 J. Perrin u. Mlle. Choucroun, C. R. hebd. Séances Acad. Sci. 184, 1097 [1927]; 189, 1213 [1929]. 
<smiles>C[Al]1CC(N)CCC1C1CCC(N)CC1</smiles>

Trypaflavin

Absorptionsmaximum 4600 A

Blaugrüne Fluoreszenz

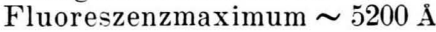

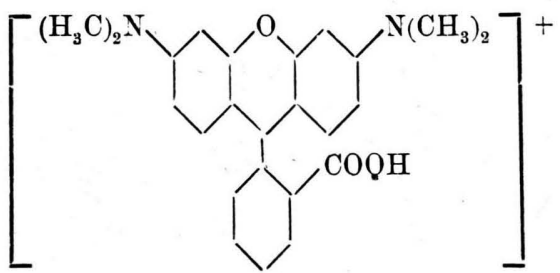

Rhodamin B

Absorptionsmaximum 5550 A

Orange Fluoreszenz

Fluoreszenzmaximum $\sim 5800 \AA$

Absorptionsmessungen im Konzentrationsbereich von $1 \cdot 10^{-5}$ bis $5 \cdot 10^{-4} \mathrm{Mol} / l$ ergaben die Gültigkeit des Beerschen Gesetzes für Trypaflavin. Bei Rhodamin B verschiebt sich dagegen beim Übergang von der konzentrierten zur verdünnten Lösung das Absorptionsspektrum um etwa $50 \AA$ zu längeren Wellen. Diese Veränderung, die auf der Dissoziation der Carboxylgruppe des Rhodamins beruht, kann durch Zugabe von Wasserstoffionen vermieden werden. Bei allen Messungen wurde deshalb den Lösungen Salzsäure in einer Konzentration von $0,003 \mathrm{Mol} / l$ zugesetzt. Für die Mischlösung beider Farbstoffe im Molverhältnis 1:1, die für die Messungen ausschließlich benutzt wurde, ist das konzentrationsunabhängige $\mathrm{Ab}$ sorptionsspektrum in Abb. 1 dargestellt ${ }^{3}$.

Die Fluoreszenzmessungen wurden visuell mit der in der früheren Veröffentlichung ausführlicher beschriebenen Anordnung durchgeführt, deren Prinzip hier nur kurz angedeutet sei.. Die Elektrode einer Punktlichtlampe wird mittels Linsen und totalreflek-

${ }^{3} \mathrm{E}_{\mathcal{B}}$ ist hier der „molare“ Extinktionskoeffizient $\varepsilon=\frac{1}{c d} \log \frac{I 0}{I}$ aufgetragen, wobei $I_{0}$ und $I$ die Lichtintensitäten vor und hinter der durchstrahlten Schicht, $d$ deren Dicke und $c$ die für beide Stoffe gleiche molare Konzentration in der Lösung ist. Diese etwas ungewöhnliche Definition ist im vorliegenden Falle der üblichen vorgezogen, in der für $c$ die Gesamtkonzentration beider Stoffe einzusetzen wäre. Der molare Extinktionskoeffizient ist in $\mathrm{cm}^{2} / \mathrm{mMol}$ $\left[=(\mathrm{Mol} / l)^{-1} \mathrm{~cm}^{-1}\right]$ angegeben. tierender Prismen auf die Oberflächen zweier Küvetten abgebildet, welche die $\mathrm{zu}$ vergleichenden Lösungen enthalten. Die dort im Fluoreszenzlicht erscheinenden Bilder werden ihrerseits auf die beiden Eintrittsblendenöffnungen eines Martensschen Polarisationsphotometers abgebildet, das den quantitativen Vergleich der Fluoreszenzintensitäten gestattet. Zur Erregung und Beobachtung gelangen dabei Lichtbündel geringer Öffnung, die annähernd senkrecht durch die Küvettenoberfläche hindurchtreten. Im Strahlengang des Erregungslichtes befindet sich ein Blaufilter, das im wesentlichen nur Licht unterhalb $4700 \AA$ durchläßt und deshalb vorwiegend das kürzerwellig absorbierende Trypaflavin erregt. Im Strahlengang des Fluoreszenzlichtes befindet sich ein Blaugrünfilter mit einer langwelligen Durchlässigkeitsgrenze bei etwa $5600 \AA \AA$, welches das Licht der Trypaflavin-

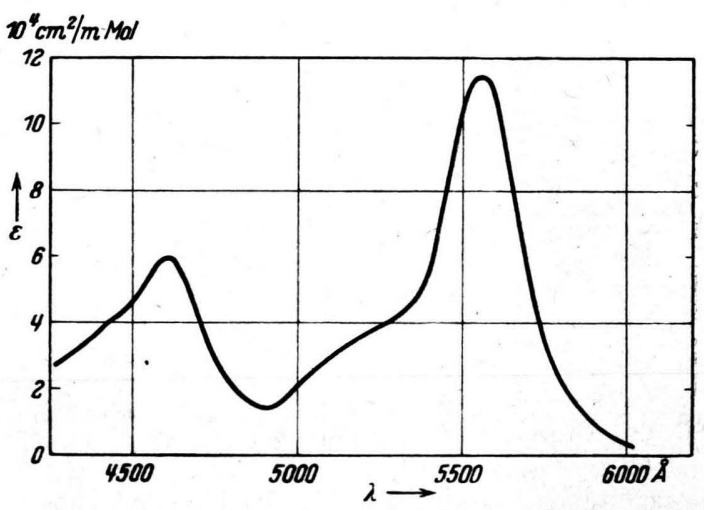

Abb. 1. Absorptionsspektrum äquimolarer Mischlösun gen von Trypaflavin und Rhodamin B in MethanolSalzsäure $(0,003-n$.) bei Konzentrationen zwischen $1 \cdot 10^{-5}$ und $5 \cdot 10^{-4} \mathrm{Mol} / l$.

fluoreszenz durchläßt, aber dasjenige der Rhodaminfluoreszenz zurückhält. Abweichend von der früher benutzten Anordnung befinden sich die Küvetten in Wasserbädern, die durch eingekittete Spiegelglasscheiben Erregung und Beobachtung gestatten. Diese Bäder erfüllen neben der hier weniger wichtigen Temperierung den Zweck, die Grenzfläche Glas-Luft von der Küvettenoberfläche zu verlegen, wodurch die Störungen durch reflektiertes Primärlicht sehr gering gehalten werden können.

Es wurden zwei zylindrische Küvetten 1 und 2 benutzt, deren Schichtdicken durch Auswägen zu 0,121 bzw. $1,015 \mathrm{~cm}$ bestimmt wurden. Das Verhältnis der Schichtdicke von Küvette $1 \mathrm{zu}$ derjenigen vọn Küvette 2 ist hiernach 0,119. Eine davon unabhängige Bestimmung durch Extinktionsmessungen wäßriger Lösungen von Kaliumferricyanid ergab unter Annahme der Gültigkeit des Beerschen Gesetzes ${ }^{4}$ den Wert 0,120 für das Schichtdickenverhältnis. Von den zu untersuchenden äquimolaren Lösungen der Farbstoffe Trypaflavin und Rhodamin B in Methanol-Salzsäure. wurde jeweils eine Lösung bestimmter Konzentration

4 Nach G. K o r tü m, Z. physikal. Chem., Abt. B, 33, 243 [1936], ist dieses hier streng gültig. 


\begin{tabular}{|c|c|c|c|c|c|c|c|c|}
\hline$c_{1}$ & . $\quad c_{2}$ & $I_{1} / I_{2}$ & $\xi$ & $g(\xi)$ & $f(\xi)$ & $I_{1}^{0} / I_{2}^{0}$ & $\begin{array}{c}\eta_{i}^{\prime} \eta_{0} \\
(\exp .)\end{array}$ & $\begin{array}{c}\eta / \eta_{0} \\
\text { (theor.) }\end{array}$ \\
\hline $\begin{array}{l}2,0 \cdot 10^{-5} \\
4,0 \cdot 10^{-5} \\
6,3 \cdot 10^{-5} \\
1,0 \cdot 10^{-4} \\
2,0 \cdot 10^{-4} \\
4,0 \cdot 10^{-4} \\
1,0 \cdot 10^{-3}\end{array}$ & $\begin{array}{r}2,4 \cdot 10^{-6} \\
4,8 \cdot 10^{-6} \\
7,55 \cdot 10^{-5} \\
1,2 \cdot 10^{-5} \\
2,4 \cdot 10^{-5} \\
4,8 \cdot 10^{-5} \\
1,2 \cdot 10^{-4}\end{array}$ & $\begin{array}{l}1,061 \\
1,033 \\
1,040 \\
1,013 \\
0,935 \\
0,807 \\
0,601\end{array}$ & $\begin{array}{c}0,38 \\
0,77 \\
1,21 \\
1,92 \\
3,8 \\
7,7 \\
19\end{array}$ & $\begin{array}{l}0,47 \\
0,44 \\
0,40 \\
0,35 \\
0,24 \\
0,13 \\
0,05\end{array}$ & $\begin{array}{l}0,946 \\
0,950 \\
0,954 \\
0,960 \\
0,973 \\
0,985 \\
0,994\end{array}$ & $\begin{array}{r}1,004 \\
0,981 \\
0,992 \\
0,973 \\
0,910 \\
0,795 \\
0,598\end{array}$ & $\begin{array}{l}1,00 \\
0,98 \\
0,99 \\
0,97 \\
0,91 \\
0,78 \\
0,57\end{array}$ & $\begin{array}{l}0,99 \\
0,97 \\
0,96 \\
0,94 \\
0,89 \\
0,79 \\
0,57\end{array}$ \\
\hline
\end{tabular}

Tab. 1. Versuchsergebnisse. $c_{1}, c_{2}$ molare Konzentration jedes Stoffes in Küvette 1 bzw. 2, $I_{1} / I_{2}$ Verhältnis der für diese gemessenen Fluoreszenzintensitäten, $\xi$ Absorptionsparameter nach Gl. $(4), g(\xi), f(\xi)$ Korrekturgrößen nach Gln. (3) und (3a), $I_{1} \mathbf{0} / I_{2}{ }^{0}$ Verhältnis der korrigierten Fluoreszenzintensitäten, $\eta / \eta_{0}$ Verhältnis der Fluoreszenzausbeute bei der Konzentration $c_{1}$ zu derjenigen bei der Konzentration 0 .

$c$ in Küvette 1 mit einer solchen der Konzentration $0,120 c$ in Küvette 2 verglichen. Die Elimination einer evtl. vorhandenen Unsymmetrie der optischen Anordnung geschah in üblicher Weise durch Vertauschen der Küvetten, wobei deren Vorderflächen an die gleichen Stellen gebracht wurden. Die so gemessenen Verhältnisse der Fluoreszenzintensitäten zwischen der konzentrierten Lösung in Küvette 1 und der verdünnteren Lösung in Küvette $\mathbf{2}$ sind in Tab. 1 angegeben ${ }^{5}$.

Es erscheint zunächst überraschend, daß die Intensitätsverhältnisse bei den unter geringen Konzentrationen ausgeführten Messungen größer als 1 sind. Unter der Annahme, daß diese Verhältnisse gleich denjenigen der inneren Fluoreszenzausbeuten seien, würde dies deren Zunahme mit wachsender Konzentration, also das Gegenteil einer Löschung, bedeuten. Wie jedoch oben bereits bemerkt, gilt die Proportionalität von Fluoreszenzintensität und innerer Fluoreszenzausbeute auch bei gleich absorbierenden Schichten nur für parallelen Strahlengang des erregenden Lichtes und des Fluoreszenzlichtes. Bei der benutzten Versuchsanordnung ist - zur Erreichung genügender Intensität - diese Bedingung keineswegs erfüllt, sondern es sind im Gegenteil Erregungslichtquelle und Blendenöffnung des Photometers durch konvergente Strahlenbündel auf die Küvettenoberflächen abgebildet. Bei einem solchen Strahlengang ist die wirksame Photometeröffnung für die in tiefer Schicht erzeugte Fluoreszenz geringer als für die an der Oberfläche erzeugte; deshalb nimmt der zur Messung gelangende Teil der Fluoreszenzintensität mit zunehmender Tiefe der fluoreszierenden Schicht ab. Bei parallelem Strah-

5 Die Werte sind, obwohl sie Mittel aus etwa zehn Einzelablesungen unter sehr günstigen photometrischen Bedingungen darstellen, höchstens auf $1 \%$ genau. Die letzte Dezimale ist mit angeführt, um unnötige Fehler bei den nachfolgenden Umrechnungen $\mathrm{zu}$ vermeiden. lengang gleiche Fluoreszenzintensitäten in verschieden dicken Küvetten erscheinen daher hier ungleich.

Durch Messung an einer Küvette geringer Schichtdicke wurde festgestellt, daß die meßbare Fluoreszenzintensität $I$ annähernd linear mit der Tiefe $x$ der Schicht abnimmt. Es ist also

$$
I=I^{0}(1-a x),
$$

wo $I^{0}$ die Fluoreszenzintensität bei Erregung in der Oberflächenschicht $x=0$ ist. Die Konstante $\alpha$ wurde für die benutzte Anordnung $\mathrm{zu} 0,13 \mathrm{~cm}^{-1}$ bestimmt.

In entsprechender Weise kann auch die in einer Küvette mit der Schichtdicke $d$ erzeugte Fluoreszenzintensität durch Ansatz der folgenden Beziehung auf die Oberflächenschicht reduziert werden:

$$
I=I^{0}[1-a d g(\xi)]
$$

Dabei ist $\xi$ ein noch genauer zu definierender Parameter, der die Absorptionsverhältnisse in der Küvette beschreibt. Bei sehr starker Absorption $(\xi=\infty)$ ist $g(\infty)=0$ und deshalb $I=I^{0}$. Die Fluoreszenz entsteht hier bereits dicht unter der Oberfläche, so daß sich die Reduktion erübrigt. Bei sehr geringer Absorption $(\xi=0)$ ist $g(0)=1 / 2$, weil die Schichten vor und hinter der Mittelebene $x=d / 2$ gleich stark emittieren. Eine unschwer durchzuführende theoretische Behandlung der Absorptionsverhältnisse in der Lösung ergibt für die Funktion $g(\xi)$ folgenden analytischen Ausdruck:

$$
g(\xi)=\frac{1-(1+\xi) e^{-\xi}}{\xi\left(1-e^{-\xi}\right)} .
$$

Der Absorptionsparameter $\xi$ ist dabei definiert durch 


$$
\xi=\ln 10 \cdot\left(\varepsilon_{\mathrm{e}}+\varepsilon_{\mathrm{f}}\right) \cdot c d,
$$

wo $c$ die molare Konzentration jedes der beiden Stoffe ist, und $\varepsilon_{\mathrm{e}}$ bzw. $\varepsilon_{\mathrm{f}}$ die molaren dekadischen Extinktionskoeffizienten ihrer Mischung für erregendes Licht und Fluoreszenzlicht bedeuten. Gl. (3) ist streng nur unter der Voraussetzung gültig, daß enge Spektralbereiche für Erregung und Beobachtung der Fluoreszenz benutzt werden. Für die vorliegenden Messungen trifft dies zwar nicht zu, doch ist der Einfluß der hieraus entstehenden Unsicherheit auf die Größe der anzubringenden Korrektur so gering, daß er unberücksichtigt bleiben kann.

Die Absorption der Lösung für das Erregungslicht wurde aus der Schwächung der Fluoreszenz berechnet, die beim Anbringen einer entsprechenden Schicht in dessen Strahlengang hervorgerufen wird. Weil dieses Licht nicht monochromatisch ist, hängen die so für $\varepsilon_{\mathrm{e}}$ erhaltenen Werte allerdings von der optischen Schichtdicke der absorbierenden Lösung ab. So ergab sich für eine $1 \mathrm{~cm}$ dicke Schicht einer Lösung mit je $5 \cdot 10^{-6} \mathrm{Mol} / l$ beider Farbstoffe $\varepsilon_{\mathrm{e}}=3,3 \cdot 10^{4} \mathrm{~cm}^{2} / \mathrm{mMol}$, für eine solche mit $1 \cdot 10^{-4} \mathrm{Mol} / l \varepsilon_{\mathrm{e}}=1,5 \cdot 10^{4} \mathrm{~cm}^{2} / \mathrm{mMol}$. Analoge Messungen im Strahlengang des Fluoreszenzlichtes ergaben für die gleichen Lösungen $\varepsilon_{\mathrm{f}}=3,5 \cdot 10^{4}$ bzw. $1,7 \cdot 10^{4} \mathrm{~cm}^{2} / \mathrm{mMol}$. Die durch diese Konzentrationsabhängigkeit des mittleren Extinktionskoeffizienten bedingte Unsicherheit in der Größe der anzubringenden Korrektur ist nur gering. Da es richtig erscheint, die bei geringer optischer Dicke erhaltenen Werte zu bevorzugen, wurde $\varepsilon_{\mathrm{e}}+\varepsilon_{\mathrm{f}}=7,0 \cdot 10^{4} \mathrm{~cm}^{2} / \mathrm{mMol}$ und, dem entsprechend, $\xi=1,6 \cdot 10^{5} c d$ angenommen. Die hiermit nach den Gln. (3) und (4) berechneten Werte von $\xi$ und $g(\xi)$ sind in Tab. 1 eingetragen. Dort sind auch die Korrekturfaktoren

$$
f=\frac{1-a d_{2} g(\xi)}{1-a d_{1} g(\xi)}
$$

angegeben, mit welchen man nach Gl. (2) das Intensitätsverhältnis $I_{1} / I_{2}$ multiplizieren muß, um das auf die Oberfläche reduzierte Intensitätsverhältnis $I_{1}{ }^{0} / I_{\Sigma}{ }^{0} \mathrm{zu}$ erhalten. Die folgende Spalte enthält die Werte dieses reduzierten Intensitätsverhältnisses selbst. Diese sind gleich den Verhältnissen der Fluoreszenzausbeute $\eta$ für die betreffenden Konzentrationen. Da die so für geringe Konzentrationen erhaltenen Werte sehr nahe an 1 liegen, erscheint es gerechtfertigt, unterhalb
$1 \cdot 10^{-5} \mathrm{Mol} / l \quad \eta$ als konstant anzunehmen und gleich dem Grenzwert $\eta_{0}$ zu setzen. Die auf dieser Grundlage rekursiv berechneten Werte von $\eta_{1} / \boldsymbol{r}_{0}$ sind in der vorletzten Spalte von Tab. 1 eingetragen. Die gleichen Werte sind auch in Abb. 2 als Funktion der Konzentration $c$ des Rhodamins (die im vorliegenden Falle gleich derjenigen des Trypaflavins ist) aufgetragen. Im Bereich hoher Konzentrationen sind die Messungen durch solche aus der früheren Veröffentlichung ergänzt. Die in Abb. 2 dargestellte Kurve gibt den Konzentrationsverlauf der Löschung nach einer ebenfalls früher bereits angegebenen Formel, deren theoretische Begründung hier noch nachzutragen ist.

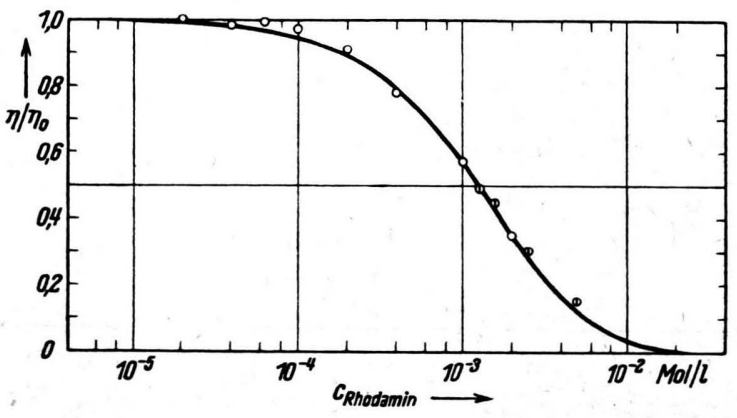

Abb. 2. Wahre Fluoreszenzlöschung von Trypaflavin in Methanol durch Rhodamin B. Relative innere Fluoreszenzausbeute als Funktion der Rhodaminkonzentration (logarithmischer Maßstab). $\bigcirc$ Neue Messungen, (1) frühere Messungen bei höheren Konzentrationen.

Es wird angenommen, daß der hier untersuchte Fall von Löschung durch Abwanderung der Elektronenanregungsenergie von Molekülen des Stoffes A zu solchen des Stoffes B zustandekomme. Aus der Theorie des zugrunde liegenden Elementarprozesses ${ }^{6}$ ist $\mathrm{zu}$ entnehmen, daß die Übergangshäufigkeit der sechsten Potenz des Molekülabstandes umgekehrt proportional ist. Dabei ist sie auf Abstände bis über $50 \AA$ mit der Ausstrahlungshäufigkeit vergleichbar. Die hierdurch bedingte Wanderung der Anregungsenergie übertrifft die Brownsche Translationsbewegung, deren mittlere Verschiebung während der Anregungsdauer für Moleküle der betrachteten Art nur etwa $20 \AA$ beträgt. Hier soll - obwohl dies nur bedingt $\mathrm{zu}$ rechtfertigen ist - die Brownsche Translationsbewegung der Moleküle überhaúpt vernachlässigt werden, so daß also die Molekül-

${ }^{6}$ Th. F örste r, Naturwiss. 33, 166 [1946]; Ann. Physik (6) 2, 55 [1948]. 
abstände während der Anregungsdauer als konstant anzunehmen sind.

Es sei ein beliebiges Molekül der Sorte A (mit kürzerwelliger Absorption und deshalb höherer Anregungsenergie) betrachtet, das zur Zeit $t=0$ gerade angeregt worden ist. Wenn keine Moleküle der Sorte B vorhanden wären, so würde es nach einer mittleren Anregungsdauer $\tau_{0}$ durch Strahlung und innermolekulare Löschprozesse desaktiviert. Seine natürliche Desaktivierungshäufigkeit (Zahl dieser Prozesse in der Zeiteinheit für ein dauernd angeregtes Molekül) ist also gleich $1 / \tau_{0}$. Ein beliebiges Molekül $k$ der Sorte B (mit längerwelliger Absorption und deshalb geringerer Anregungsenergie), das sich im Abstand $R_{k}$ von dem angeregten befindet, gibt diesem eine weitere Möglichkeit zur Desaktivierung. Deren Häufigkeit ist unter Berücksichtigung der oben angegebenen Abstandsabhängigkeit gleich $\frac{1}{\tau_{0}}\left(\frac{R_{0}}{R_{k}}\right)^{6}$ zu setzen. Die hier eingeführte Länge $R_{0}$ ist gleich demjenigen Abstand, für welchen die Energieübergangshäufigkeit gerade gleich der natürlichen Desaktivierungshäufigkeit ist. In dieser Weise ist die Möglichkeit des Energieüberganges von dem betrachteten Molekül A zu sämtlichen Molekülen $\mathrm{B}$ zu berücksichtigen, während der umgekehrte Übergang (von einem Molekül mit geringerer zu einem solchen mit höherer Anregungsenergie) vernachlässigt werden kann. Dies führt zu folgender Differentialgleichung für die zeitliche Abnahme der Anregungswahrscheinlichkeit @ $(t)$ des betrachteten Moleküls A:

$$
-\frac{d \varrho}{d t}=\frac{1}{\tau_{0}}+-\frac{1}{\tau_{0}} \sum_{k=1}^{N}\left(\frac{R_{0}}{R_{k}}\right)^{6} .
$$

Dabei sei $N$ die Zahl der Moleküle B in einem endlichen Volumen $V$ der Lösung. Die Integration von Gl. (5) mit der Anfangsbedingung $\varrho(0)=1$ ergibt

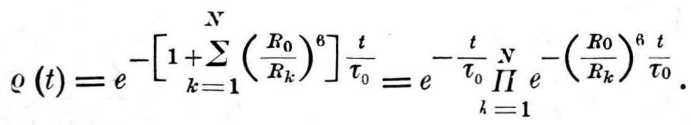

Die Anregungswahrscheinlichkeit des betrachteten Moleküls A klingt hiernach rein exponentiell ab. Tatsächlich gelangt aber nicht der Abklingvorgang des Einzelmoleküls, sondern derjenige einer Gesamtheit von Molekülen mit statistisch verteilten Abständen $R_{k}$ zur Beobachtung. Sei $w(R) d R$ die Wahrscheinlichkeit, ein bestimmtes Molekül $\mathrm{B}$ in der Umgebung des angeregten mit einem Abstand zwischen $R$ und $R+d R$ anzutreffen, so ist die Abnahme der mittleren Anregungswahrscheinlichkeit $\overline{\varrho(t)}$ folgendermaßen darzustellen:

$$
\begin{aligned}
\overline{\varrho(t)}=e^{-\frac{t}{\tau_{0}}} \underset{\prod_{k=1}^{N} \int_{0}^{R_{g}}-\left(\frac{R_{0}}{R_{k}}\right)^{6} \frac{t}{\tau_{0}}}{ } v\left(R_{k}\right) d R_{k} \\
=e^{-\frac{t}{\tau_{0}}}[J(t)]^{N},
\end{aligned}
$$

mit

$$
J(t)=\int_{0}^{R_{g}} e^{-\left(\frac{R_{0}}{R}\right)^{6} \frac{t}{\tau_{0}}} w(R) d R .
$$

Es ist dabei angenommen, daß das Volumen $V$ eine Kugel mit dem angeregten Molekül A als Mittelpunkt und $R_{\mathrm{g}}$ deren Radius sei:

$$
V=\frac{4 \pi}{3} R_{\mathrm{g}}^{3} .
$$

Die Verteilungsfunktion $w(R)$ hängt im Prinzip von den zwischen den Molekülen A und B wirkenden Kräften ab, wobei vor allem an solche elektrostatischer Natur wegen deren großer Reichweite zu denken ist. Da aber selbst deren Reichweite kleiner als $R_{0}$ ist, mögen auch diese unberücksichtigt bleiben ${ }^{7}$. Die Wahrscheinlichkeit $w(R)$ werde daher gleich derjenigen gesetzt, die sich für eine gleichmäßige Raumverteilung ergibt:

$$
w(R) d R=\frac{4 \pi R^{2} d R}{V} .
$$

Es ist dann das in Gl. (8) eingeführte Integral

$$
\begin{aligned}
J(t)=\frac{4 \pi}{V} \int_{\mathrm{o}}^{R_{\mathrm{g}}} e^{-\left(\frac{R_{\mathrm{0}}}{R}\right)^{6} \frac{t}{\tau_{0}}} & R^{2} d R \\
& =\frac{1}{2} \sqrt{\zeta_{\mathrm{g}}} \int_{\zeta_{\mathrm{g}}}^{\infty} e^{-\zeta} \frac{d \zeta}{\sqrt{\zeta^{3}}},
\end{aligned}
$$

7 Die Reichweite der elektrostatischen Kräfte in der Lösung ist auf solche Abstände $R_{\text {el }}$ begrenzt, für welche die potentielle Energie die mittlere thermische Energie pro Freiheitsgrad übertrifft. Dies führt zu einem Grenzabstand $R_{\mathrm{el}} \sim e^{2} / \varepsilon k T$, wo $e$ die Elementarladung, $\varepsilon$ die Dielektrizitätskonstante, $k$ die Boltzmannsche Konstante und $T$ die absolute Temperatur ist. Im vorliegenden Falle ist $R_{\mathrm{el}} \sim 20 \AA$. 
mit den Abkürzungen

und

$$
\zeta=\left(\frac{R_{0}}{R}\right)^{6} \frac{t}{\tau_{0}}
$$

$$
\zeta_{\mathrm{g}}=\left(\frac{R_{0}}{R_{\mathrm{g}}}\right)^{6} \frac{t}{\tau_{0}} \ll 1 .
$$

Die Ungleichung $\zeta_{\mathrm{g}} \ll 1$ gilt für alle in Betracht kommenden Zeiten $t$, weil der Wirkungsradius $R_{0}$ des Energieüberganges sehr viel kleiner als der makroskopische Radius $R_{\mathrm{g}}$ ist. Unter dieser Voraussetzung läßt sich das in Gl. (9) auftretende Integral in eine Reihe nach steigenden Potenzen von $\sqrt{\zeta_{\mathrm{g}}}$ entwickeln, deren erste Glieder folgendermaßen lauten ${ }^{8}$ :

$$
\int_{\zeta_{\mathrm{g}}}^{\infty} e^{-\zeta} \frac{d \zeta}{\sqrt{\zeta^{3}}}=\frac{2}{\sqrt{\zeta_{\mathrm{g}}}}-2 \sqrt{\pi}-6 \sqrt{\zeta_{\mathrm{g}}}+\ldots
$$

Damit ergeben die Gln. (9) und (7) unter Vernachlässigung höherer Potenzen von $\sqrt{\zeta_{\mathrm{g}}}$

$$
J(t)=1-\sqrt{\pi \zeta_{\mathrm{g}}}
$$

$$
\overline{\varrho(t)}=e^{-\frac{t}{\tau_{0}}}\left(1-\sqrt{\pi \zeta_{\mathrm{g}}}\right)^{N} .
$$

Da die Molekülzahl $N$ in dem zugrunde gelegten Flüssigkeitsvolumen $V$ sehr groß ist, begeht man keinen Fehler, indem man statt der rechten Seite von Gl. (13) deren Grenzwert für $N \rightarrow \infty$ setzt. Man erhält so unter Berücksichtigung der bekannten Definition der Konstante $e$ als Grenzwert von $(1+1 / N)^{N}$

8 Man erhält dies durch partielle Integration

$$
\begin{aligned}
& \int_{\zeta_{\mathrm{g}}}^{\infty} e^{-\zeta \zeta^{-3 / 2}} d \zeta=-\left[2 e^{-\zeta \zeta^{-1 / 2}}\right]_{\zeta_{\mathrm{g}}}^{\infty}-2 \int_{\zeta_{\mathrm{g}}}^{\infty} e^{-\zeta} \zeta^{-1 / 2} d \zeta \\
& =2 e^{-\zeta_{\mathrm{g}} \zeta_{\mathrm{g}}^{-\frac{1}{2}}}-2 \int_{\mathrm{o}}^{\infty} e^{-\zeta} \zeta^{-\frac{1}{2}} d \zeta+2 \int_{0}^{\zeta_{\mathrm{g}}} e^{-\zeta} \zeta^{-1 / 2} d \zeta .
\end{aligned}
$$

Das Integral im zweiten Glied dieser Summe ist* $\left(-\frac{1}{2}\right) !=\sqrt{\pi}$. Durch Entwicklung der Exponentialfunktion in den beiden anderen Gliedern nach Poten-

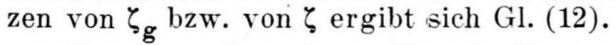

* Vgl. etwa Jahnke u. Emde, Funktionentafeln, 3. Aufl., 1938, S. 11.

$$
\begin{aligned}
\overline{O(t)} & =e^{-\frac{t}{\tau^{v}}} \lim _{N \rightarrow \infty}\left(1-\frac{1}{N} N \sqrt{\pi \zeta_{\mathrm{g}}}\right)^{N} \\
& =e^{-\frac{t}{\tau_{0}}-N \sqrt{\pi \zeta_{\mathrm{g}}}}=e^{-\frac{t}{\tau_{0}}-\frac{V \bar{\pi} N R_{\mathrm{n}}{ }^{3}}{R_{\mathrm{g}}{ }^{3}} \sqrt{\tau_{0}}} .
\end{aligned}
$$

Obwohl also der Abklingvorgang am Einzelmolekül streng exponentiell erfolgt, ist er im Mittel über die Gesamtheit der Moleküle nicht durch eine einfache Exponentialfunktion darstellbar. Aus Gl. (14) ist zu entnehmen, daß die Anregung zunächst stärker, dann schwächer abklingt, als es nach jener Formel der Fall wäre. Dies beruht darauf, daß zunächst solche Moleküle $A$, welche zufällig Moleküle B in ihrer unmittelbaren Nähe vorfinden, ihre Anregungsenergie rasch verlieren, und dann solche übrig bleiben, die ihre Anregungsenergie langsamer abgeben.

Die hier durchgeführten Messungen betreffen allerdings den Abklingvorgang nicht unmittelbar. Mit der Funktion $\overline{\mathbf{Q}(t)}$, der Wahrscheinlichkeit, ein zur Zeit $t=0$ angeregtes Molekül zur Zeit $t$ noch angeregt vorzufinden, steht aber die Fluoreszenzausbeute in Zusammenhang. Da die Zahl der. Emissionsprozesse pro Zeitintervall dieser Wahrscheinlichkeit proportional ist, ergibt sich die Fluoreszenzausbeute $\eta$ proportional deren Integral über die ganze Zeitdauer:

$$
\eta=C \int_{0}^{\infty} \overline{\varrho(t)} d t
$$

wo $C$ eine Konstante ist. Bei nicht vorhandener Löschung $(N=0)$ erhält man aus Gl. (14)

$$
\eta_{0}=C \int_{0}^{\infty} e^{-\frac{t}{\tau_{0}}} d t=C \tau_{0} .
$$

Daher ist die relative Fluoreszenzausbeute

$$
\frac{\eta}{\eta_{0}}=\frac{1}{\tau_{0}} \int_{\mathrm{o}}^{\infty} \overline{Q(t)} d t .
$$

Zur Berechnung dieses Integrals führt man zweckmäßig die dimensionslose Veränderliche $s=t / \tau_{0}$ und die Konstante

$$
q=\frac{\sqrt{\pi}}{2} \frac{N R_{0}^{3}}{R_{\mathrm{g}}{ }^{3}}
$$


ein. Es ist dann ${ }^{9}$

$$
\begin{aligned}
\eta / \eta_{0}=\int_{0}^{\infty}{ }_{0(s)} d s= & \int_{0}^{\infty} e^{-s-2 q \sqrt{s}} d s \\
& =1-\sqrt{\pi} q e^{q^{2}}[1-\Phi(q)] .
\end{aligned}
$$

Dabei ist

$\Phi(q)=\frac{2}{\sqrt{\pi}} \int_{q}^{\infty} e^{-x^{2}} d x$ das Gaußsche Fehlerintegral .

Die hier auftretende Größe $q$ ist nach ihrer Definition in Gl. (16) der Zahl $N$ der löschenden Moleküle B im Volumen $V$ und damit der molaren Konzentration $c$ des Stoffes B proportional. (Die Konzentration des Stoffes A tritt hier überhaupt nicht auf.) Setzt man dementsprechend $q=c / c_{0}$, so ergibt sich schließlich

$$
\eta / \eta_{0}=1-\sqrt{\pi}\left(c / c_{0}\right) e^{\left(c / c_{0}\right)^{2}}\left[1-\Phi\left(c / c_{0}\right)\right]
$$

Durch Reihenentwicklung erhält man die beiden Grenzfälle

$$
\begin{gathered}
\eta / \eta_{0} \sim 1-\sqrt{\pi} c / c_{0}, \quad c \ll c_{0} \\
\eta / \eta_{0} \sim c_{0}^{2} / 2 c^{2}, \quad c \gg c_{0} .
\end{gathered}
$$

Für die kritische Konzentration $c_{0}$ erhält man aus Gl. (16), indem man an Stelle von $N$ die Zahl $N^{\prime}=6,02 \cdot 10^{20}$ der Moleküle im Millimol einführt:

$$
c_{0}=\frac{3}{2 \sqrt{\pi^{3}}} \frac{1}{N^{\prime} R_{0}^{3}} .
$$

In Abb. 2 ist der nach Gl. (18) berechnete Konzentrationsverlauf von $\eta / \eta_{0}$ für den Wert $c_{0}=2,3 \cdot 10^{-3} \mathrm{Mol} / l$ eingetragen, mit welchem die beste Darstellung der Versuchsdaten erreicht wird. Die Übereinstimmung ist mindestens so gut, wie man für die hier gegebene Theorie in Anbetracht ihrer zahlreichen Vernachlässigungen

- Zur Ausführung der hier auftretenden Integration führt man die Substitution $x=\sqrt{s}+q$ durch und erhält so

$$
\begin{array}{r}
\eta / \eta_{0}=e^{q^{2}} \int_{0}^{\infty} e^{-\left(V_{s}^{-}+q\right)^{2}} d s=2 e^{q^{2}} \int_{q}^{\infty} e^{-x^{2}}(x-q) d x \\
=1-2 q e^{q^{2}} \int_{q}^{\infty} e^{-x^{2}} d x .
\end{array}
$$

erwarten darf. Bei geringen Konzentrationen scheint die Löschung etwas schwächer zu sein, als sie durch Gl. (18) dargestellt wird.

Mit $c_{0}=2,3 \cdot 10^{-3} \mathrm{Mol} / l$ berechnet man aus Gl. (19) den kritischen Abstand für den Energieübergang Trypaflavin $\rightarrow$ Rhodamin $\mathrm{zu}^{10} R_{0}=58 \AA$. Wenn auch die quantitative Theorie des zwischenmolekularen Energieüberganges nur für gleichartige Moleküle entwickelt worden ist, kann die Verallgemeinerung für verschiedenartige Moleküle leicht gegeben werden. Man erhält so

$$
R_{0}=\frac{\hat{\lambda}_{\mathrm{m}}}{2 \pi n} \sqrt{\frac{3}{8} \frac{\eta_{0}}{\tau_{0} \Delta \nu}} .
$$

Hier treten außer den oben bereits definierten GröBen $\eta_{0}$ und $\tau_{0}$ des Moleküls $\mathrm{A}$ noch die folgenden auf: $\lambda_{m}$, eine Wellenlänge zwischen dem Fluoreszenzmaximum von $\mathrm{A}$ und dem Absorptionsmaximum von $\mathrm{B}$; $n$, der Brechungsindex des Lösungsmittels und

$$
\frac{1}{\Delta v}=\frac{\int f_{\mathrm{A}}(v) \cdot \varepsilon_{\mathrm{B}}}{\int f_{\mathrm{A}}(v) d v \cdot \int \varepsilon_{\mathrm{B}}(v) d v} .
$$

$1 / \Delta \nu$ ist das normierte Überdeckungsintegral des Fluoreszenzspektrums $f_{\mathrm{A}}(v)$ von $\mathrm{A}$ und des $\mathrm{Ab}$ sorptionsspektrums $\varepsilon_{B}(v)$ von $B$ auf der Frequenzskala. Die Überdeckung dieser Spektren ist die wesentliche Voraussetzung für einen Energieübergang der betrachteten Art.

Obwohl die Daten für eine quantitative Prüfung von Gl. (20) nicht vorliegen, ist doch leicht festzustellen, daß diese die richige Größenordnung für $R_{0}$ liefert. Mit $\lambda_{\mathrm{m}}=5300 \AA, n=1,33, \eta_{0} \sim 1$, $\tau_{0} \sim 1 \cdot 10^{-8} \mathrm{sec}$ und $\Delta \nu \sim 3 \cdot 10^{12} \mathrm{sec}^{-1}$ (ungefähre Halbwertsbreite d.Spektren) erhält $\operatorname{man} R_{0}=63 \AA$.

Aus Gl. (14) läßt sich auch die mit der Löschung verbundene Veränderung der mittleren Abklingdauer $\tau$ berechnen. Diese nimmt ab, jedoch schwächer als proportional mit der Ausbeute $\eta$. Von den üblicherweise unterschiedenen Typen der statischen und der dynamischen Löschung ist der erste mit keiner, der zweite mit einer zu $\eta$ proportionalen Abnahme der mittleren Abklingdauer $\tau$ verbunden. Der hier betrachtete Fall von Löschung steht also in dieser Beziehung zwischen den Typen der statischen und der dynamischen Löschung.

${ }^{10}$ In der früheren Veröffentlichung ${ }^{1}$ (S. 321) ist auf Grund einer gröberen Abschätzung ein etwas größe. rer Abstand angenommen worden. 